\title{
Spinal Cord Compression, CTCAE
}

National Cancer Institute

\section{Source}

National Cancer Institute. Spinal Cord Compression, CT CAE. NCI Thesaurus. Code

C143233.

A disorder characterized by pressure on the spinal cord. 